\title{
Further evidence supporting the allosteric regulation of PEPCK by anions.
}

\author{
S Barwell ${ }^{1}$, T Holyoak ${ }^{2}$ \\ ${ }^{1}$ University of Waterloo, Waterloo, ON, ${ }^{2}$ Biology Dept, Univ of Waterloo \\ saebarwe@uwaterloo.ca
}

Phosphoenolpyruvate carboxykinase (PEPCK) primarily catalyzes the conversion of oxaloacetic acid to phosphoenolpyruvate as one of the key steps of gluconeogenesis. However, PEPCK is known to be thermodynamically reversible in vitro, catalyzing the reverse of the gluconeogenic reaction, albeit with a lower enzyme activity. These two seemingly conflicting pieces of data pointed towards a hypothesis of kinetic inhibition of PEPCK in vivo. Previous research was presented that elucidated a potential 'reverse-direction' specific mechanism of inhibition of PEPCK. Sufficient evidence was provided for the presence of an allosteric site in PEPCK, leading to inhibition of catalysis in the reverse direction while catalytic function in the 'forward' direction was largely unaffected. Structural and kinetic data supported the model that inhibition in the form of small anions, such as chloride, binding to the allosteric site contributed to the observed unidirectionality of the PEPCK-catalyzed reaction in vivo. Previous research implemented a novel use of anomalous diffraction data to support this hypothesis. PEPCK crystals were soaked over a range of increasing iodide concentrations from 10 to $500 \mathrm{mM}$ (as a proxy for chloride) and the corresponding anomalous signals located at the allosteric site were found to titrate over the range of iodide. Other iodide binding sites including the active site were not found to titrate, and instead no relationship between iodide concentration and anomalous signal could be determined. Binding isotherms were generated for the various binding sites and the resulting binding constant determined for the allosteric site was comparable to the competitive inhibition constant from the kinetic data using chloride. Expanding upon these initial data we have undertaken additional studies to support the allosteric model of anion regulation of PEPCK that include the creation of allosteric site mutant PEPCK forms. Structural and kinetic data collected on these mutants similar to that which was previously collected on the WT enzyme provide further support for our model of directional allosteric regulation of PEPCK by monovalent anions. To further support the model of allosteric regulation we are undertaking studies to directly determine the binding of chloride ions to PEPCK rather than using iodide as a proxy by collecting anomalous diffraction data at long wavelengths.

Acta Cryst. (2020). A76, a18 